\title{
Mad revival of cancer
}

\author{
Hong Liu ${ }^{1}$, Hongtao $\mathrm{Yu}^{1}$ \\ ${ }^{\prime}$ Department of Pharmacology, Howard Hughes Medical Institute, University of Texas Southwestern Medical Center at Dallas, \\ 6001 Forest Park Rd, Dallas, TX75390, USA \\ Cell Research (2010) 20:394-396. doi:10.1038/cr.2010.38; published online 1 April 2010
}

Aneuploidy (wrong numbers of chromosomes) is a hallmark of cancer cells and arises from chromosome missegregation in mitosis. To prevent aneuploidy, cells employ surveillance systems to monitor mitosis. The spindle checkpoint (also known as the mitotic checkpoint) is one such surveillance system conserved from yeast to man $[1,2]$. During each mitosis, this checkpoint detects aberrant kinetochoremicrotubule attachments, inhibits the anaphase-promoting complex or cyclosome (APC/C), stabilizes cyclin B1 and securin, and delays anaphase onset until all sister chromatids reach proper microtubule attachment. Mad2 is a critical player of the spindle checkpoint and contributes to the inhibition of $\mathrm{APC} / \mathrm{C}$ directly [3]. Targeted partial inactivation of the spindle checkpoint genes, including Mad2, results in aneuploidy and cancer predisposition in mice [4]. Mutations of BubR1, another spindle checkpoint gene whose product is a direct APC/C inhibitor, cause mosaic variegated aneuploidy (MVA) syndrome, a rare human genetic disease [5]. Patients with MVA are prone to develop various cancers, among other phenotypes, indicating that BubR1 is a tumor suppressor in humans.

Despite the vital roles of the spindle checkpoint in maintaining chromosome

\footnotetext{
Correspondence: Hongtao Yu

Tel: +1-214-645-6161

E-mail: Hongtao.Yu@utsouthwestern.edu
}

stability and the widespread aneuploidy seen in cancer cells, mutations of the spindle checkpoint genes are surprisingly rare in human cancers. The underlying reasons for this observation are unknown, but it has been suggested that drastic reduction of the spindle checkpoint activity might result in gross chromosome missegregation, which causes organismal or even cell lethality, as opposed to cancer progression [1]. Therefore, subtle perturbations of the spindle checkpoint, such as altered expression of checkpoint genes, may be necessary for cells to acquire tolerable levels of aneuploidy that can produce certain abnormal karyotypes conducive to cancer formation. For example, Mad2 is a target gene of the E2F transcription factor [6]. As a consequence, Mad2 is often overexpressed in human tumors lacking the retinoblastoma $(\mathrm{Rb})$ tumor suppressor, which is an E2F inhibitor [6]. Mad2 overexpression at levels much higher than the endogenous levels is indeed sufficient to initiate tumor in multiple tissues in mice [7]. Consistently, cells from these Mad2 transgenic mice exhibit broken chromosomes, anaphase bridges, and aneuploidy. Interestingly, high levels of Mad2 are not required for tumor maintenance in these mice, suggesting that $\mathrm{CIN}$ as a consequence of Mad2 overexpression, but not $\operatorname{Mad} 2$ itself, is the culprit responsible for cancer formation in these animals.

On the other hand, human cancers do not express Mad2 at such high levels. Can modest Mad2 overexpression contribute to tumorigenesis? How does dysfunction of the spindle checkpoint collaborate with other oncogenic events? Do CIN and aneuploidy play roles in other stages of tumorigenesis? A recent study by Sotillo et al. [8] has begun to address these questions by investigating the interplay between Mad2 overexpression and the oncogenic Ras pathway in lung cancer in mice. In this study, Sotillo et al. created transgenic mice that express Mad2 or an oncogenic Ras mutant $\left(\operatorname{Kras}^{\mathrm{G} 12 \mathrm{D}}\right)$ or both driven by doxycycline-inducible promoters in the lung. The expression level of the Mad2 transgene is comparable to that of the endogenous Mad2. Mad2 overexpression at these moderate levels was not sufficient to drive lung cancer formation, but it cooperated with $\mathrm{Kras}^{\mathrm{G} 12 \mathrm{D}}$ to produce more aggressive, genetically heterogeneous tumors.

Cancer cells often continue to depend on the activities of certain cancerinitiating oncogenes for survival, a phenomenon termed oncogene addiction. Sotillo et al. further tested whether Mad2 overexpression affected Kras oncogene addiction of the tumors in the transgenic mice expressing $\mathrm{Kras}^{\mathrm{G} 12 \mathrm{D}}$ alone or expressing both Mad2 and $\mathrm{Kras}^{\mathrm{G} 12 \mathrm{D}}$ [8]. Removal of doxycycline from their diet turned off ectopic expression of both Mad2 and $\mathrm{Kras}^{\mathrm{G} 12 \mathrm{D}}$ in these animals and caused tumor regression in both groups of mice, indicating that Mad2 overexpression was not sufficient 
to completely alleviate the oncogene addiction of tumors initially triggered by Ras activation. Remarkably, tumors recurred in about $50 \%$ of the mice expressing both $\mathrm{Mad} 2$ and $\mathrm{Kras}^{\mathrm{G} 12 \mathrm{D}}$ within a year of doxycycline removal whereas none of the mice expressing $\mathrm{Kras}^{\mathrm{G} 12 \mathrm{D}}$ alone relapsed in this time window. The recurrent tumors were morphologically and genetically heterogeneous and most had lost the expression of the Mad2 or Kras ${ }^{\mathrm{G} 12 \mathrm{D}}$ transgenes, suggesting that these tumors developed different strategies to eventually overcome their addiction to the Ras pathway. Therefore, Mad2 overexpression promotes tumor relapse after the inactivation of tumor-initiating oncogenes. Their findings provide a possible explanation for why Mad2 overexpression is common in human cancers.

How does transient Mad2 overexpression contribute to tumor relapse? Sotillo et al. suggested that Mad2 overexpression led to a hyperactive spindle checkpoint and CIN [8]. Although the mechanism by which a hyperactive spindle checkpoint produced CIN was not established, their results were consistent with CIN playing a major role in tumor recurrence in the transgenic mice. How then does CIN promote tumor relapse? Sotillo et al. further proposed that CIN might produce more genetic diversity within the cancer cell population or enhance the rate of mutations or both [8]. This could allow a subset of these cancer cells to acquire additional oncogenic mutations or lose tumor suppressors, which were no longer dependent on the initial oncogenic pathway for survival.

If CIN indeed contributes to tumor relapse, one interesting issue that remains unresolved is the actual sequence of events. In one model (Figure 1A), CIN-mediated genetic diversity in cancer cells occurs prior to oncogene withdrawal. A small subset of these cells is already independent of the initiating oncogenic pathway for viability. Upon oncogene withdrawal, most cancer cells
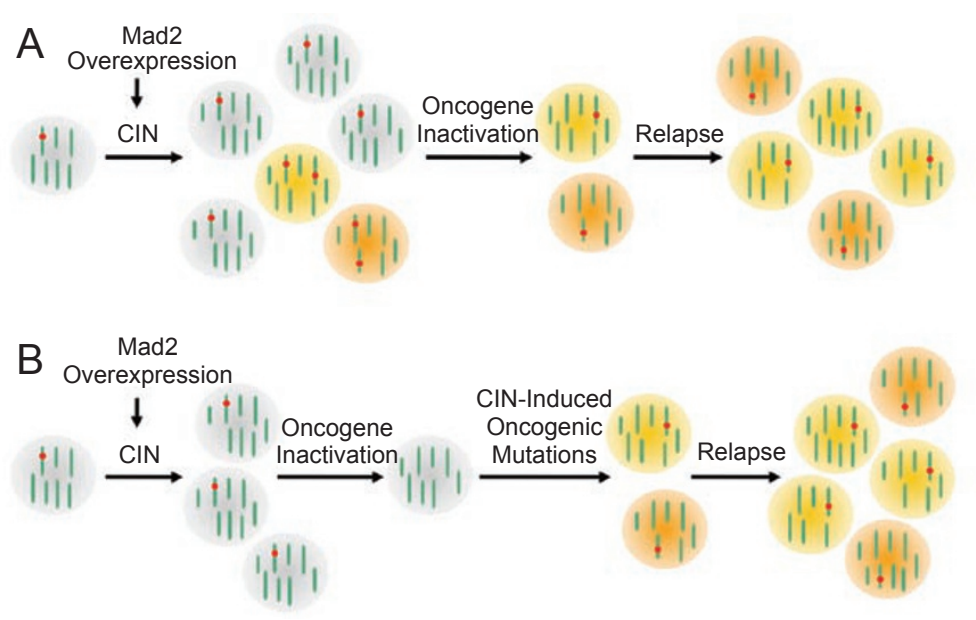

Figure 1 Models for CIN-induced tumor relapse following oncogene inactivation. Green bars and red dots signify chromosomes and oncogenic mutations, respectively. See text for details.

die, leading to drastic tumor regression. The small surviving population then expands, causing cancer relapse. In the second model (Figure 1B), all cancer cells are still dependent on the initial oncogenic pathway for survival, despite the fact that they exhibit CIN and are genetically heterogeneous. Upon oncogene withdrawal, this population of cells is under strong selective pressure. Cells with a dysfunctional spindle checkpoint have a higher probability of acquiring additional oncogenic mutations and evolve to become independent of the initial oncogenic pathway. Expansion of these cells then causes cancer relapse. A simple experiment to test these models is to construct transgenic mice that express Mad2 and $\mathrm{Kras}^{\mathrm{G} 12 \mathrm{D}}$ under different inducible promoters. After tumors form in these mice, the Mad2 transgene is turned off for an extended period before $\mathrm{Kras}^{\mathrm{G} 12 \mathrm{D}}$ is turned off. If tumors fail to recur in these mice, this would support the second model.

Relapse is a major problem in the treatment of human cancers. Patients who respond to targeted cancer therapies often relapse and develop more aggressive cancers that no longer respond to the original treatment. As stated above, Mad2 is commonly overexpressed in human cancers. Other mechanisms of spindle checkpoint dysregulation in human cancers have been reported [9]. A dysfunctional spindle checkpoint could be a mechanism for cancer relapse in human patients. If a dysfunctional spindle checkpoint only provides an evolutionary advantage for cancer cells to escape oncogene addiction after oncogene inactivation (the second model mentioned above), then restoration of proper spindle checkpoint function in cancer cells prior to targeted oncogene inactivation therapy might reduce incidences of cancer relapse.

\section{References}

1 Bharadwaj R, Yu H. The spindle checkpoint, aneuploidy, and cancer. Oncogene 2004; 23:2016-2027.

2 Musacchio A, Salmon ED. The spindleassembly checkpoint in space and time. Nat Rev Mol Cell Biol 2007; 8:379-393.

3 Yu H. Regulation of APC-Cdc20 by the spindle checkpoint. Curr Opin Cell Biol 2002; 14:706-714.

4 Michel LS, Liberal V, Chatterjee A, et al. MAD2 haplo-insufficiency causes premature anaphase and chromosome instability in mammalian cells. Nature 2001; 409:355-359.

5 Hanks S, Coleman K, Reid S, et al. Constitutional aneuploidy and cancer 
predisposition caused by biallelic mutations in BUB1B. Nat Genet 2004; 36:1159-1161.

6 Hernando E, Nahle Z, Juan G, et al. Rb inactivation promotes genomic instability by uncoupling cell cycle progression from mitotic control. Nature 2004;
430:797-802.

7 Sotillo R, Hernando E, Diaz-Rodriguez $\mathrm{E}$, et al. $\mathrm{Mad} 2$ overexpression promotes aneuploidy and tumorigenesis in mice. Cancer Cell 2007; 11:9-23.

8 Sotillo R, Schvartzman JM, Socci ND, Benezra R. Mad2-induced chromo- some instability leads to lung tumour relapse after oncogene withdrawal. Nature 2010 Feb 21. doi:10.1038/nature 08803

9 Qi W, Yu H. The spindle checkpoint and chromosomal stability. Genome Dyn 2006; 1:116-130. 